\title{
Biosynthesis of chiral 3-hydroxyvalerate from single propionate-unrelated carbon sources in metabolically engineered $E$. coli
}

\author{
Hsien-Chung Tseng ${ }^{1}$, Catey L Harwell ${ }^{1}$, Collin H Martin ${ }^{1,2,3}$, Kristala LJ Prather ${ }^{1,2^{*}}$
}

\begin{abstract}
Background: The ability to synthesize chiral building block molecules with high optical purity is of considerable importance to the fine chemical and pharmaceutical industries. Production of one such compound, 3-hydroxyvalerate $(3 \mathrm{HV})$, has previously been studied with respect to the in vivo or in vitro enzymatic depolymerization of biologically-derived co-polymers of poly(3-hydroxybutyrate-co-3-hydroxyvalerate). However, production of this biopolymeric precursor typically necessitates the supplementation of a secondary carbon source (e.g., propionate) into the culture medium. In addition, previous approaches for producing 3HV have not focused on its enantiopure synthesis, and thus suffer from increased costs for product purification.

Results: Here, we report the selective biosynthesis of each $3 \mathrm{HV}$ stereoisomer from a single, renewable carbon source using synthetic metabolic pathways in recombinant strains of Escherichia coli. The product chirality was controlled by utilizing two reductases of opposing stereoselectivity. Improvement of the biosynthetic pathway activity and host background was carried out to elevate both the $3 \mathrm{HV}$ titers and $3 \mathrm{HV} / 3 \mathrm{HB}$ ratios. Overall, shake-flask titers as high as $0.31 \mathrm{~g} / \mathrm{L}$ and $0.50 \mathrm{~g} / \mathrm{L}$ of $(S)-3 \mathrm{HV}$ and $(R)-3 \mathrm{HV}$, respectively, were achieved in glucose-fed cultures, whereas glycerol-fed cultures yielded up to $0.19 \mathrm{~g} / \mathrm{L}$ and $0.96 \mathrm{~g} / \mathrm{L}$ of $(S)-3 \mathrm{HV}$ and $(R)-3 \mathrm{HV}$, respectively.

Conclusions: Our work represents the first report of direct microbial production of enantiomerically pure $3 \mathrm{HV}$ from a single carbon source. Continued engineering of host strains and pathway enzymes will ultimately lead to more economical production of chiral $3 \mathrm{HV}$.
\end{abstract}

\section{Background}

The efficient production of enantiomerically pure chemicals from renewable resources has gained considerable attention especially in the fine chemical/pharmaceutical industry. Stereo-selective chemical processes generally employ expensive chiral catalysts, require harsh physical conditions and solvents, and suffer from extensive byproduct formation. In contrast, enzyme-catalyzed reactions are highly stereo-selective and can be performed in aqueous solutions under mild conditions [1]. As a result, replacing chemical processes by biological ones for the synthesis of chiral compounds has been extensively investigated not only due to superior stereoselectivity of enzymatic reactions but also due to

\footnotetext{
* Correspondence: kljp@mit.edu

'Department of Chemical Engineering, Massachusetts Institute of Technology, Cambridge, MA 02139, USA

Full list of author information is available at the end of the article

sustainability as an implementation of green chemistry [2-5]. One example is the production of hydroxyacids, a family of versatile chiral molecules containing one hydroxyl group and one carboxyl group [6]. These molecules have the potential to serve as useful chiral building blocks for a diverse range of products, including polyhydroxyalkanoates (PHAs) (biodegradable polymers) and optically-active fine chemicals, such as pharmaceuticals, vitamins, antibiotics, and flavor compounds [7-10]. Naturally, hydroxyacids are primarily found to be polymerized as PHAs where they serve as intracellular storage materials for numerous microbes. Those PHAs consist mostly of monomers with 3-hydroxy, 4-hydroxy, and 5-hydroxy groups with different lengths of main and side chains [11].

Among the hydroxyacid monomers, 3-hydroxybutyrate $(3 \mathrm{HB})$ is the most prolific, with several reports on engineering $E$. coli for its production from renewable 
feedstocks [5,12-15]. Biosynthesis of 3HB begins with the condensation of two acetyl-CoA molecules, a commonly found cellular metabolite regardless of carbon source (Figure 1). However, economically-feasible production of longer-chain hydroxyacids is complicated by issues such as low yields and high prices of feedstocks due to the need to supplement a second carbon source. One example of such hydroxyacids is 3-hydroxyvalerate $(3 \mathrm{HV})$. The production of $3 \mathrm{HV}$ has been realized by the hydroxylation of valeric acid through fermentation of Candida rugosa [16]. It has also been reported that 3-hydroxyvaleronitrile can be converted into $3 \mathrm{HV}$ using the nitrilase activity of Comamonas testosteroni [17]. More recently, direct biological production of $3 \mathrm{HV}$ was demonstrated using recombinant $P$. putida KT2440 and levulinic acid as substrate, although the levulinic acid metabolism pathway in P. putida KT2440 has not yet been fully elucidated [18]. In the aforementioned cases, valeric acid, 3-hydroxyvaleronitrile, and levulinic acid were supplied as secondary carbon sources (in addition to glucose). Additionally, the chirality and/or enantiopurity of the $3 \mathrm{HV}$ produced in the above-mentioned studies is unclear as they did not report whether the synthesized $3 \mathrm{HV}$ was in the $R, S$, or racemic form. Alternatively, $3 \mathrm{HV}$ can be obtained through either the in vivo or in vitro enzymatic depolymerization of synthesized poly(3-hydroxybutyrate-co-3-hydroxyvalerate) (PHBV), a well known biodegradable polymer marketed as Biopol ${ }^{\mathrm{Tm}}$ which is produced by the natural PHA accumulating bacterium Ralstonia eutropha when grown on glucose and propionate [19]. The production of PHBV has also been reported in recombinant $E$. coli upon introduction of the PHA biosynthesis genes of $R$. eutropha and when grown in glucose medium supplemented with valine or threonine [20]. Regardless of whether the end product is $3 \mathrm{HV}$ or $\mathrm{PHBV}$, it can be generally concluded that supplementation of a second carbon source, such as valeric acid, 3-hydroxyvaleronitrile, levulinic acid, propionate, valine, or threonine in addition to glucose, is necessary to provide the 5-carbon unit precursor of $3 \mathrm{HV}$. Unfortunately, the high price and/or toxicity of the added second carbon sources could limit industrial production of $3 \mathrm{HV}$ [21]. Therefore, synthesis of $3 \mathrm{HV}$ from a single carbon source has been proposed as an efficient and sustainable avenue in contrast to the above-mentioned systems.

A novel pathway for the production of PHBV solely from glycerol has been established in recombinant Salmonella enterica Serovar Typhimurium, containing a heterologous pathway that converts succinyl-CoA to propionyl-CoA, the essential precursor molecule of $3 \mathrm{HV}$ CoA in PHBV synthesis [22]. However, expensive cyanocobalamin $\left(\mathrm{CN}-\mathrm{B}_{12}\right)$ was supplemented to the medium to provide the precursor of coenzyme $B_{12}$ required for the activity of one of the enzymes in the $\mathrm{B}_{12}$-dependent biosynthetic pathway. It should also be noted that the pathway only functioned in S. enterica, a pathogen, but not $E$. coli, thus limiting its applicability to other industriallyrelevant host organisms. In this study, we proposed an alternative biosynthetic pathway that does not require coenzyme $\mathrm{B}_{12}$ for its functionality to synthesize $3 \mathrm{HV}$ from glucose or glycerol. Specifically, we metabolically engineered $E$. coli to exploit its native metabolism for endogenous supply of propionyl-CoA via the threonine biosynthesis pathway, and introduced a heterologous pathway for chiral 3HV biosynthesis using acetyl-CoA and propionyl-CoA as precursor molecules. As stated above, several previous methods for producing $3 \mathrm{HV}$ did not focus on enantiopure synthesis. Similarly, due to the stereospecific constraints of PHBV synthesis, in which polymers are composed exclusively of $(R)-3 \mathrm{HB}$ and $(R)-3 \mathrm{HV}$ monomer units, the synthesis of $(S)$-3HV from PHBV remains effectively impossible. On the contrary, our proposed pathway makes possible the direct synthesis of both enantiomerically pure $(R)-3 \mathrm{HV}$ and $(S)-3 \mathrm{HV}$.

We have identified a pathway which combines elements of our previously developed chiral 3HB biosynthesis pathway together with the natural threonine biosynthesis pathway of E. coli for direct biosynthesis of chiral 3HV (Figure 1 ). In the proposed pathway, chiral $3 \mathrm{HV}$ is produced from direct hydrolysis of $3 \mathrm{HV}-\mathrm{CoA}$ catalyzed by a thioesterase II (encoded by tes $B$ ) where $3 \mathrm{HV}-\mathrm{CoA}$ is obtained from condensation of one acetyl-CoA and one propionyl-CoA to form 3-ketovaleryl-CoA catalyzed by a thiolase (encoded by $b k t B$ ), followed by a reduction of the 3 ketovaleryl-CoA to $3 \mathrm{HV}-\mathrm{CoA}$ catalyzed by a 3-hydroxybutyryl-CoA dehydrogenase. Here, two enantio-selective 3-hydroxybutyryl-CoA dehydrogenases were utilized to control the chirality of $3 \mathrm{HV}-\mathrm{CoA}$ produced. The NADPHdependent dehydrogenase encoded by phaB produces $(R)$ $3 \mathrm{HV}-\mathrm{CoA}$ while the NADH-dependent dehydrogenase encoded by $h b d$ produces $(S)-3 \mathrm{HV}-\mathrm{CoA}$. It should be noted that in order to yield the highest $3 \mathrm{HV}$ titers and $3 \mathrm{HV} / 3 \mathrm{HB}$ ratios, BktB was used as the thiolase in this study as opposed to other thiolases such as PhaA from $R$. eutropha $\mathrm{H} 16$ or Thil from C. acetobutylicum ATCC 824 because BktB has been shown to have highest in vitro enzyme activity towards the $C_{5}$ substrate while PhaA and Thil were specific towards the $C_{4}$ substrate [19]. Next, a pathway allowing for endogenous propionyl-CoA synthesis from glucose or glycerol, through the threonine metabolic pathway intermediate 2-ketobutyrate, was introduced to circumvent the need for feeding propionate. To examine the upstream pathway for endogenous supply of propionyl-CoA, we used a bottom-up approach where 2-ketobutyrate and threonine were, at first, fed to provide propionyl-CoA, in addition to glucose, to support $3 \mathrm{HV}$ production. In the final stage, a single carbon source of 


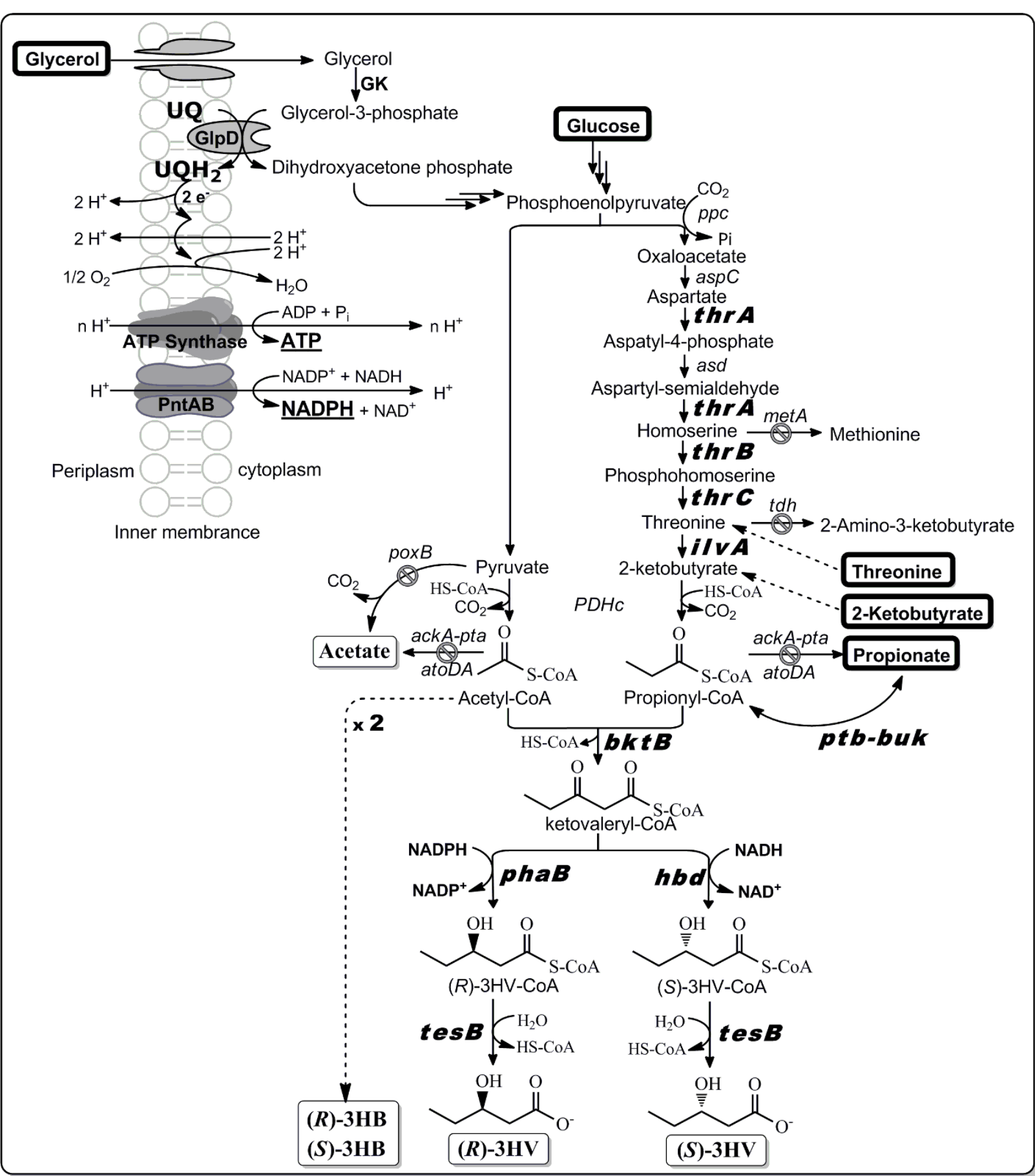

Figure 1 Schematic representation of chiral $3 \mathrm{HV}$ production via the threonine biosynthesis pathway in metabolically engineered E. coli. Genes in bold are overexpressed while disrupted pathway steps are indicted by the "no" symbols. The carbon sources and main metabolic products in the system are enclosed by rectangular boxes with thick and thin lines, respectively. For glycerol utilization [43,44], a glycerol kinase (GK) phosphorylates glycerol to glycerol-3-phosphate, followed by oxidation to dihydroxyacetone phosphate that enters glycolysis. The oxidation reaction is catalyzed by a membrane enzyme called glycerol-3-phosphate dehydrogenase (GlpD) with concomitant production of ubiquinol $\left(\mathrm{UQH}_{2}\right)$ from ubiquinone $(\mathrm{UQ})$. Electrons stored in the ubiquinol are then transferred through the aerobic respiratory chain coupled with proton translocation from cytoplasm to periplasm. Both ATP and NADPH can be synthesized by an $\mathrm{H}^{+}$-driven proton movement from periplasm to cytoplasm, catalyzed by an ATP synthase and a membrane-bound transhydrogenase (PntAB), respectively. 
glucose or glycerol was used to provide both acetyl-CoA and propionyl-CoA to support 3HV biosynthesis in our metabolically engineered $E$. coli.

Overall, in this study we successfully demonstrated the direct biological production of enantiomerically pure $(R)$-3HV and $(S)$-3HV from a single carbon source. Improvements of the biosynthetic pathway and E. coli host strains have also been carried out to elevate $3 \mathrm{HV}$ titers and $3 \mathrm{HV} / 3 \mathrm{HB}$ ratios.

\section{Results}

\section{HV Synthesis from Glucose and propionate}

Acetyl-CoA is an obligate central intermediate occurring in any organism and under any physiological condition; however, this is not the case for propionyl-CoA, which is only synthesized under special physiological conditions and from only few substrates [23]. Therefore, synthesis of $3 \mathrm{HV}-\mathrm{CoA}$ requires propionyl-CoA biosynthesis. To validate our $3 \mathrm{HV}$ biosynthesis pathway, propionate was initially fed to provide propionyl-CoA as a precursor molecule to ensure the downstream pathway was capable of making chiral 3HV. It has been reported that the R. eutropha PHA biosynthesis genes can be functionally expressed in E. coli, resulting in homopolymer PHB production from glucose [24]. However, low levels of $3 \mathrm{HV}$ monomer within the synthesized co-polymer PHBV was observed in recombinant $E$. coli when propionate was co-fed with glucose in a way analogous to the procedure used for $R$. eutropha [24]. One explanation for the low content of $3 \mathrm{HV}$ monomer is that E. coli does not possess an efficient system for importing and/or converting propionate to propionyl-CoA. Therefore, to address the propionate utilization problem, a CoA-activation mechanism (encoded by the $p t b-b u k$ operon [25]) was incorporated into our previously developed 3HB pathway to investigate the substrate elasticity of the pathway for $3 \mathrm{HV}$ production.

Our results show that, in the absence of the CoA-activation mechanism, i.e. Ptb-Buk, only trace amount of $3 \mathrm{HV}$ was produced (Figure 2). On the contrary, introducing Ptb-Buk into the pathway yielded up to $2 \mathrm{~g} / \mathrm{L}$ of both enantiomers of $3 \mathrm{HV}$. It was noted that for strains expressing Ptb-Buk but leaving out TesB, only $(R)$ hydroxyacids (when PhaB was employed) were produced, consistent with a previous report that Ptb-Buk forms a reversible, stereo-seletive enzyme system [13]. Overall, these results indicate that CoA-activation was crucial for propionate utilization and, most importantly, all enzymes originally utilized for $3 \mathrm{HB}$ biosynthesis were able to catalyze synthesis of $\mathrm{C}_{5}$ molecules.

\section{HV Synthesis from Glucose and 2-Ketobutyrate}

Propionyl-CoA can also be produced from 2-ketobutyrate, a common keto-acid intermediate for isoleucine

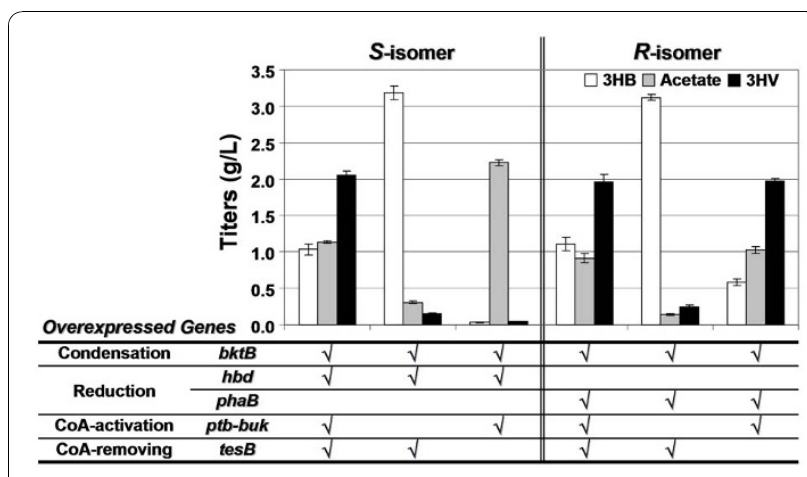

Figure 2 3HV biosynthesis from glucose and propionate. This figure shows shake-flask production of chiral $3 \mathrm{HV}$ by recombinant E. coli strain HCT 10 grown in LB supplemented with $20 \mathrm{~g} / \mathrm{L}$ glucose and $20 \mathrm{mM}$ sodium propionate. Over-expressed genes are indicated in the table below the graph.

biosynthesis, by the action of the endogenous pyruvate dehydrogenase complex enzyme (encoded by $P D H c$ ) (Figure 1) [26]. We first compared 3HV production from glucose and 2-ketobutyrate using pathways with and without over-expression of the $p t b$-buk operon. The results showed that the presence of Ptb-Buk reduced production of propionate (only observed in the $R$-isomer construct) and $3 \mathrm{HB}$ while increasing production of acetate and $3 \mathrm{HV}$, yielding $(S)-3 \mathrm{HV}$ and $(R)-3 \mathrm{HV}$ with titers up to $0.38 \mathrm{~g} / \mathrm{L}$ and $1.02 \mathrm{~g} / \mathrm{L}$, respectively (Figure 3 ). The increased production of acetate and $3 \mathrm{HV}$ was presumably due to the promiscuous activity of Ptb-Buk on cleaving excess acetyl-CoA and activating excess propionate. Given that $3 \mathrm{HB}$ production is a second-order reaction that should have a rate proportional to the square of the concentration of acetyl-CoA, a reduced acetylCoA pool resulting from the promiscuous activity of

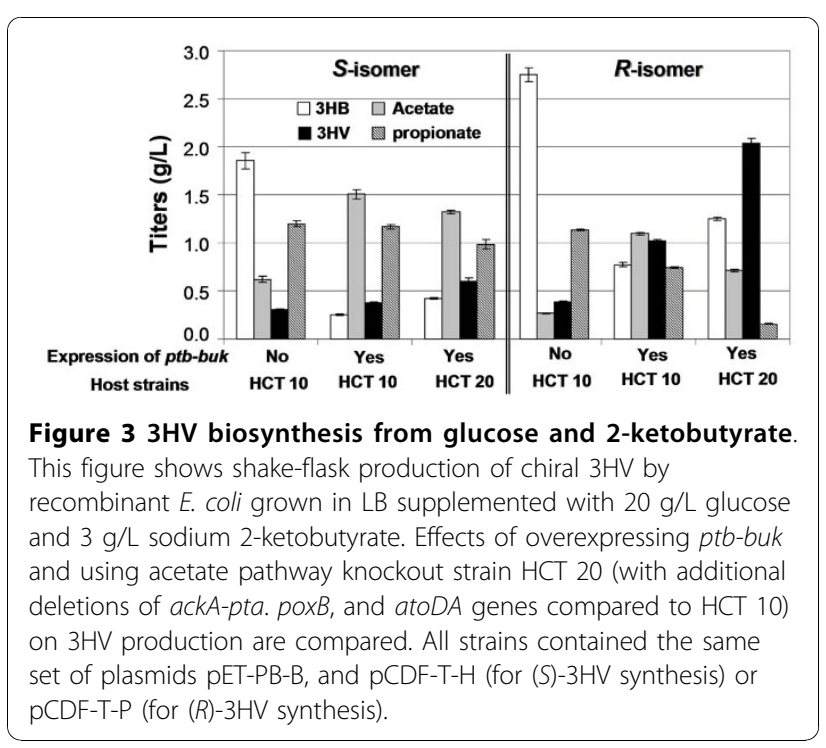


Ptb-Buk likely caused a significant decrease in $3 \mathrm{HB}$ production. In addition, propionyl-CoA is a competing substrate for $\mathrm{BktB}$, so an increase in propionyl-CoA concentration may also reduce $3 \mathrm{HB}$ production.

In an effort to decrease acetate and increase $3 \mathrm{HV}$ production, several genes, including atoDA (encoding acetoacetyl-CoA transferase), pox $B$ (encoding pyruvate oxidase), and ackA-pta (encoding acetate kinase and phosphate acetyltransferase) were deleted, and the resulting strain was designated as HCT 20 . The production of $(S)-3 \mathrm{HV}$ and $(R)-3 \mathrm{HV}$ was further boosted to titers of $0.60 \mathrm{~g} / \mathrm{L}$ and $2.04 \mathrm{~g} / \mathrm{L}$, respectively, in the recombinant acetate pathway knockout strains (HCT 20). In general, those strains produced less acetate and propionate and yielded more $3 \mathrm{HB}$ and $3 \mathrm{HV}$ compared to strains without these mutations (based on HCT 10), probably due to preserved acetyl-CoA and propionylCoA pools as a result of the introduced mutations. An empty-plasmid control experiment has also been conducted in the strain HCT 20 (that was not introduced with the $3 \mathrm{HV}$ pathway), yielding only trace amounts of acetate and propionate when grown in LB supplemented with glucose and 2-ketobutyrate (data not shown). This indicates that the production of acetate and propionate in the recombinant HCT 20 was attributed to the introduced CoA-cleaving activity conferred by $p t b-b u k$ and tesB.

\section{HV Synthesis from Glucose and Threonine}

The metabolic intermediate 2-ketobutyrate can be produced from threonine by the action of threonine deaminase. Co-feeding of threonine with glucose, together with over-expression of $E$. coli threonine deaminase (encoded by $i l v A$ ), was able to achieve production of $(S)$-3HV and $(R)-3 \mathrm{HV}$ with titers up to $0.11 \mathrm{~g} / \mathrm{L}$ and $0.22 \mathrm{~g} / \mathrm{L}$, respectively (Figure 4 ). Given that $E$. coli threonine deaminase is subject to feedback inhibition by isoleucine, a feedback resistant gene from Corynebacterium glutamicum [27] was also used, and the production of $(S)-3 \mathrm{HV}$ and $(R)-3 \mathrm{HV}$ was further boosted to titers of $0.27 \mathrm{~g} / \mathrm{L}$ and $0.91 \mathrm{~g} / \mathrm{L}$, respectively, under the same culture conditions. This experiment has also been conducted in the recombinant acetate pathway knockout strains (HCT 20); however, no improvement in production of $3 \mathrm{HB}$ and $3 \mathrm{HV}$ was observed (data not shown).

\section{HV Synthesis from Glucose}

We have demonstrated the production of chiral 3HV from glucose supplemented with propionate, 2-ketobutyrate, or threonine, in recombinant E. coli. The next step is to construct a threonine over-producing strain in an attempt to achieve $3 \mathrm{HV}$ biosynthesis from a single carbon source. To do so, we up-regulated the threonine biosynthesis pathway by over-expressing the thr $A B C$

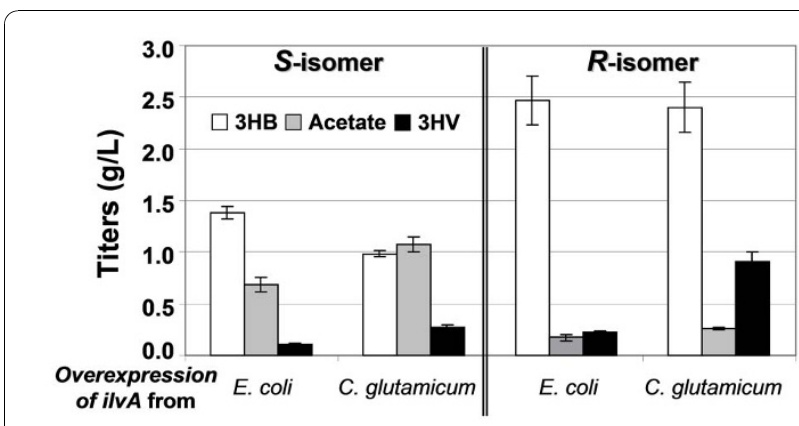

Figure 4 3HV biosynthesis from glucose and threonine. This figure shows shake-flask production of chiral $3 \mathrm{HV}$ by recombinant E. coli strain HCT 10 grown in LB supplemented with $20 \mathrm{~g} / \mathrm{L}$ glucose and $3 \mathrm{~g} / \mathrm{L}$ threonine. Chiral $3 \mathrm{HV}$ production using alternative threonine deaminases (encoded by ilvA) from E. coli and C. glutamicum is compared. All strains contained the same set of plasmids pET-PB-B, pCOLA-lcg or pCOLA-lec as indicated, and pCDFT-H (for (S)-3HV synthesis) or pCDF-T-P (for (R)-3HV synthesis).

opeon, cloned from the wild type $E$. coli or the threonine producer E. coli ATCC 21277 that has a single amino acid alteration in the homoserine dehydrogenase (encoded by $t h r A^{\text {G1297A }}$ ) for relieved feedback-inhibition [28]. Transcriptional attenuation of those genes was removed by replacing the native promoter with a T7lac promoter, allowing for IPTG-inducible expression. In addition, the pathways that compete with threonine formation as well as degrade threonine were eliminated by knocking out metA (encoding homoserine O-succinyltransferase) and $t d h$ (encoding threonine dehydrogenase) genes, yielding strain HCT 21 .

Our results showed that there was essentially no difference in $3 \mathrm{HV}$ production between strains expressing the wild type and feedback resistant thrA (data not shown) probably because threonine did not accumulate or its level was not high enough to exert a feedback inhibition to ThrA. We also compared $3 \mathrm{HV}$ production across three different $E$. coli strains, including HCT 10, HCT 20, and HCT 21. The mutants HCT 20 and HCT 21 carrying only empty plasmids significantly reduced acetate production to $0.22 \mathrm{~g} / \mathrm{L}$ as opposed to $1.85 \mathrm{~g} / \mathrm{L}$ by HCT 10 (Figure 5); however, recombinant mutant HCT 20 or HCT 21 containing the $3 \mathrm{HV}$ pathway produced as much acetate as the recombinant HCT 10, a counterintuitive finding (see Discussion). The deletions of $m e t A$ and $t d h$ enhanced (S)-3HV production by $41 \%$ (recombinant HCT 21 relative to recombinant HCT 20), but essentially had no effect on $(R)-3 \mathrm{HV}$ production. Nevertheless, those mutations were able to boost the ratios of $3 \mathrm{HV} / 3 \mathrm{HB}$ by decreasing the $3 \mathrm{HB}$ titers and/or increase the $3 \mathrm{HV}$ titers. Overall, titers as high as $0.31 \mathrm{~g} / \mathrm{L}$ and $0.50 \mathrm{~g} / \mathrm{L}$ of $(S)-3 \mathrm{HV}$ and $(R)-3 \mathrm{HV}$ were achieved in the recombinant HCT 21 with $3 \mathrm{HV} / 3 \mathrm{HB}$ ratios up to 0.35 and 0.24 , respectively (Figure 5). 


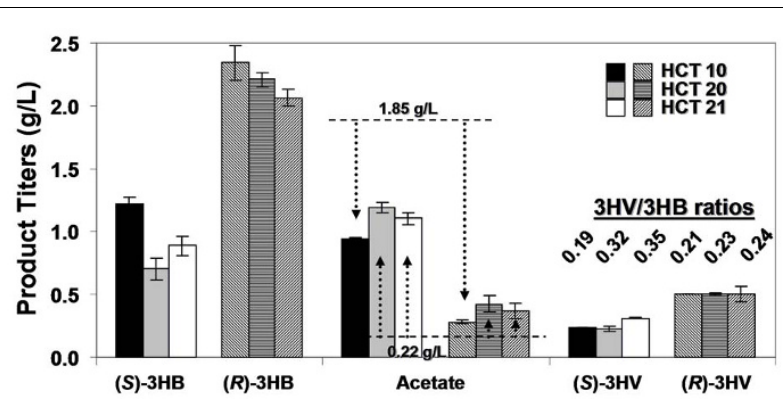

Figure 5 3HV biosynthesis solely from glucose. This figure shows shake-flask production of chiral $3 \mathrm{HV}$ in various knock-out strains as described in Table 1. Cells were grown in LB supplemented with $20 \mathrm{~g} / \mathrm{L}$ glucose. The top and bottom dashed lines represent the acetate titers produced from E. coli strain HCT 10 and HCT 20 harboring empty plasmids, respectively. All strains contained the same set of plasmids PET-PB-B, pCOLA-Tecm-lcg, and pCDF-T-H (for (S)-3HV synthesis) or pCDF-T-P (for (R)-3HV synthesis). The recombinant HCT 10 strains carrying an empty pCOLAduet- 1 in place of pCOLA-Tecm-Icg, as control strains, produced essentially no 3HV (data not shown)

\section{HV Synthesis from Glycerol}

Glycerol has become a promising and abundant carbon source due to its generation as an inevitable byproduct of biodiesel production from vegetable oils or animal fats through a transesterification reaction [29]. There have been several reports on converting glycerol to more valuable compounds such as thymidine, ethanol, and 1,3-propanediol [30-32]. Glycerol is also more reduced than glucose, leading to a higher reduced cofactor pool in the cytoplasm [32]. Therefore, in addition to glucose, we investigated the ability of our recombinant E. coli to convert glycerol to chiral 3HV. Titers of 0.08 $\mathrm{g} / \mathrm{L}$ and $0.96 \mathrm{~g} / \mathrm{L}$ of $(S)-3 \mathrm{HV}$ and $(R)-3 \mathrm{HV}$, respectively, were achieved in recombinant HCT 10, while recombinant HCT 21 produced $0.19 \mathrm{~g} / \mathrm{L}$ and $0.60 \mathrm{~g} / \mathrm{L}$ of $(S)$ $3 \mathrm{HV}$ and $(R)-3 \mathrm{HV}$, respectively (Figure 6$)$. As mentioned in the Materials and Methods section, in this specific experiment, concentration of $3 \mathrm{HB}$ was quantified by DAD at $210 \mathrm{~nm}$ that had a detection limit at around $0.08 \mathrm{~g} / \mathrm{L}$. As a result, the amounts of $(S)$-3HB produced in both recombinant HCT 10 and HCT 21 strains were too low to be quantified so that we could not report the $3 \mathrm{HV} / 3 \mathrm{HB}$ ratios. Nonetheless, in the case of $(R)$-isomers, $3 \mathrm{HV} / 3 \mathrm{HB}$ ratios could be as high as 0.88 and 1.10 , respectively, in recombinant HCT 10 and HCT 21 strains. The high $3 \mathrm{HV} / 3 \mathrm{HB}$ ratios can be beneficial in terms of product separation or biosynthesis of PHBV that enables high $3 \mathrm{HV}$ content.

\section{Confirmation of 3HV Stereochemistry}

The stereochemistry of the resulting $3 \mathrm{HV}$ in the media from these cultures was determined by methyl esterification of the $3 \mathrm{HV}$ present followed by chiral HPLC

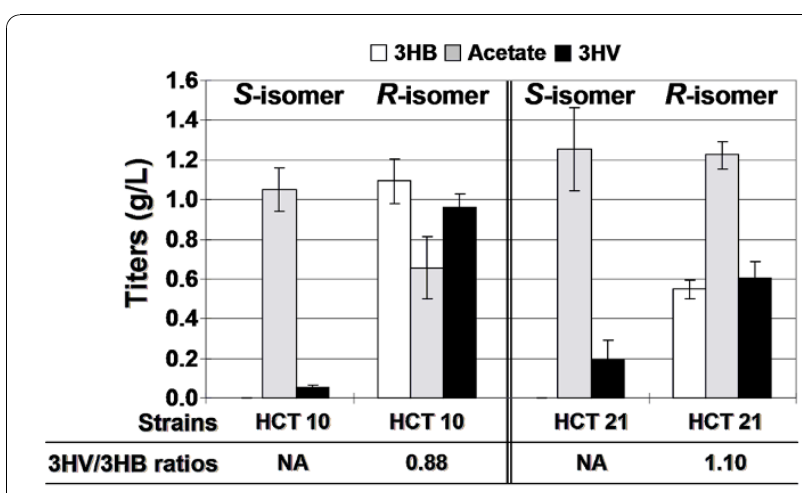

Figure $6 \mathbf{3 H V}$ biosynthesis solely from glycerol. This figure shows shake-flask production of chiral $3 \mathrm{HV}$ in various knock-out strains as described in Table 1. Cells were grown in LB supplemented with $20 \mathrm{~g} / \mathrm{L}$ glycerol. The amounts of (S)-3HB produced in both recombinant HCT 10 and HCT 21 strains were too low to be quantified due to a low detection limit by DAD at 210 $\mathrm{nm}$; therefore, the $3 \mathrm{HV} / 3 \mathrm{HB}$ ratios were not applicable (NA) to the (S)-isomer. All strains contained the same set of plasmids PET-PB-B, pCOLA-Tecm-lcg, and pCDF-T-H (for (S)-3HV synthesis) or pCDF-T-P (for (R)-3HV synthesis).

analysis using our previously developed method [15] However, we could not assign an absolute stereochemistry to each sample due to the unavailability of enantiopure $3 \mathrm{HV}$ standards. However, based on our previous results regarding the product stereochemistry of $p h a \mathrm{~B}$ and $h b d$ and the observation that $\mathrm{Me}-(R)-3 \mathrm{HB}$ has a faster retention time relative to $\mathrm{Me}-(S)-3 \mathrm{HB}$, we expect $\mathrm{Me}-(R)-3 \mathrm{HV}$ to have a faster retention time than Me$(S)$-3HV when analyzed by the same method. Thus, the 6.9 and 9.2 min peaks likely represent $\mathrm{Me}-(R)-3 \mathrm{HV}$ and $\mathrm{Me}-(S)-3 \mathrm{HV}$, respectively (Figure 7$)$. These results confirm the enantiopurity of biosynthesized 3HV.

\section{Discussion}

In general, two approaches can be taken to engineer E. coli for direct $3 \mathrm{HV}$ production via the threonine biosynthesis pathway. The first is to utilize an existing threonine producer, such as E. coli ATCC 21277 [33], followed by further engineering to introduce our constructed $3 \mathrm{HV}$ pathway. However, this and other available threonine producing strains have typically been developed through multiple rounds of random mutagenesis and selection due to the difficulty of engineering this highly regulated and complex metabolic network. Although there are several successful cases in developing industrial threonine producers by such approaches, resultant strains usually also suffer from undesired phenotypes including, for example, growth retardation, low transformation efficiency, and by-product formation as a result of random mutations [34]. In addition, other uncharacterized mutations may hinder further strain development as often needed. Fortunately, recent 


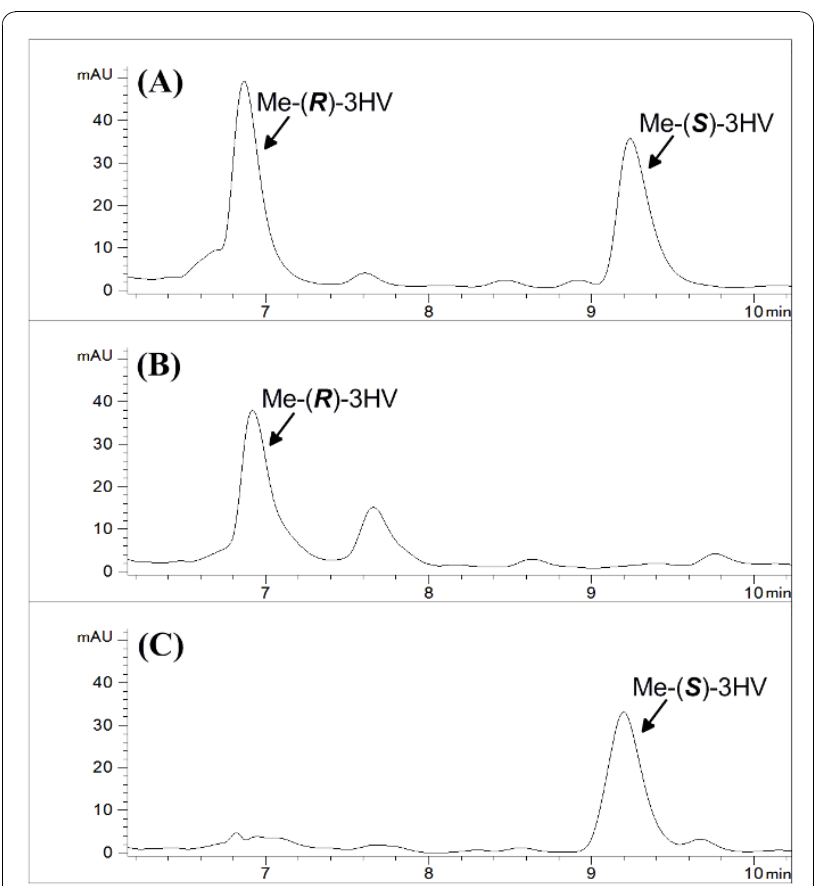

Figure 7 Determination of the stereochemistry of $3 \mathrm{HV}$. HPLC spectra of (A) racemic $3 \mathrm{HV}$ standards after boiling in methanol, (B) culture medium from the recombinant strain HCT 10 expressing $b k t B$, phaB, tesB, and ptb-buk after boiling in methanol, and (C) culture medium from the recombinant strain HCT 10 expressing $b k t B, h b d$, tesB, and ptb-buk after boiling in methanol are shown.

advances in computational genomics have allowed for rational development of production strains [34]. Therefore, as a second approach, a genetically-defined threonine producing strain was established and introduced with the $3 \mathrm{HV}$ pathway to achieve direct microbial production of chiral 3HV from glucose or glycerol.

As seen in Figure 5 acetate is the major byproduct to the production of hydroxyacids ( $3 \mathrm{HB}$ and $3 \mathrm{HV}$ ). In an effort to decrease acetate and increase $3 \mathrm{HV}$ production, a mutant strain HCT 20 with deletions on atoDA, poxB, and ackA-pta genes was developed. Counter-intuitively, the recombinant acetate pathway knockout strains of HCT 20 and HCT 21 produced slightly more acetate and less $3 \mathrm{HB}$ than recombinant HCT 10 . We suspected that the enzymatic activity responsible for acetate production was restored by Ptb-Buk and TesB in the recombinant HCT 20 and HCT 21. In fact, in a separate experiment, both enzymes were found to have CoAremoving activities on acetyl-CoA and propionyl-CoA (data not shown), so an introduction of TesB and/or Ptb-Buk to strains HCT 20 or HCT 21 would likely restore the ability to produce acetate.

Apparently, knocking out enzymes responsible for acetate production failed to reduce acetate synthesis. Alternatively, to alleviate the substrate promiscuity of
TesB and Ptb-Buk on acetyl-CoA, and thus reduce acetate production, one approach called enzyme colocalization could be implemented to allow substrate channeling between enzymes [35]. For example, pathway enzymes of $\mathrm{Hbd}$ and $\mathrm{TesB}$, catalyzing successive reactions, can be co-localized in an attempt to reduce the amount of freely floating TesB that may hydrolyze acetyl-CoA as well as to increase accessibility of 3HVCoA by TesB. The spatial organization of the enzymes can be achieved using either the leucine zipper, a dimer resulting from interaction between leucine residues [36], or the synthetic scaffolds, constructed from protein-protein interaction domains [37]. Furthermore, expressing enzymes that would assimilate produced acetate is another way to reduce acetate accumulation. For example, acetyl-CoA synthetase (encoded by acs) from E. coli can be over-expressed to convert acetate to acetyl-CoA with the use of one ATP. While successfully demonstrated in one work [38], in our case, over-expression of acs was found to have essentially no effect on acetate reduction (data not shown). Additionally, to overcome the hurdle of acetate reduction, approaches like protein engineering of TesB and/or Ptb-Buk to alleviate their substrate promiscuity, or utilization of better isozymes with more stringent substrate specificity could also mitigate the carbon loss in the form of acetate.

Among microbes, NADH and NADPH play a central role in energy metabolism by providing the cell with the reducing power for a variety of cellular redox reactions. The availability of such cofactors could impose a huge impact on the functionality of introduced biosynthetic pathways. In fact, we have previously shown that the $\mathrm{NADPH} / \mathrm{NADP}^{+}$ratio was two- to three-fold higher than the NADH $/ \mathrm{NAD}^{+}$ratio under the culture conditions examined, presumably affecting in vivo activities of $\mathrm{PhaB}$ and $\mathrm{Hbd}$ and resulting in greater production of $(R)$-3HB than (S)-3HB [15]. Given that our proposed $3 \mathrm{HV}$ pathway was based on the previously established $3 \mathrm{HB}$ pathway, it was also expected to see the same trend of greater production of $(R)-3 \mathrm{HV}$ than $(S)-3 \mathrm{HV}$, even though the cofactor dependency of 3HV synthesis may be complicated by the energetically expensive threonine biosynthesis pathway with utilization of both ATP and NADPH. In an effort to perturb the cofactor balance within the cells, thereby tuning the production of $(R)-3 \mathrm{HV}$ and $(S)-3 \mathrm{HV}$, we attempted to used glycerol, a promising, abundant, and highly-reduced carbon source, to support $3 \mathrm{HV}$ production. Based on our calculation of reducing equivalents $\left(\mathrm{e}^{-}\right)$of glucose and glycerol, on the same basis of 2 moles of phosphoenolpyruvate synthesized, glucose and glycerol possess, respectively, 24 and 28 reducing equivalents. Potentially, the additional four reducing equivalents can be utilized to generate two NADPH or equivalent amount of ATP. 
In fact, it has been experimentally confirmed that a higher intracellular NADPH/NADP ${ }^{+}$ratio was observed when glycerol was used as a carbon source than glucose, and this higher ratio was also reflected in boosted production of thymidine as its biosynthesis requires NADPH as a cofactor [32]. Given that both NADPH and ATP play a central role in threonine biosynthesis, we hypothesized that the use of glycerol, which could generate more NADPH and ATP (Figure 1) relative to glucose, may favor threonine biosynthesis by directing more carbon flux towards production of propionyl-CoA, thus favoring the formation of $3 \mathrm{HV}$ relative to $3 \mathrm{HB}$. In agreement with our hypothesis, a higher $3 \mathrm{HV} / 3 \mathrm{HB}$ ratio was obtained in the $(R)$-3HV production when glycerol was used as the carbon source (Figure 6). In addition, much larger ratios of the total (R)-hydroxyacids (summation of (R)-3HB and (R)-3HV titers) to the total (S)hydroxyacids (summation of (S)-3HB and (S)-3HV titers) were observed in glycerol-fed cultures (Figure 6) compared to glucose-fed cultures (Figure 5). We hypothesize that the higher intracellular NADPH/NADP + ratio as a result of the use of glycerol would favor (R)hydroxyacid biosynthesis compared to the use of glucose, thus yielding the larger ratios of total (R)hydroxyacids to total (S)-hydroxyacids.

As mentioned previously, BktB was chosen as the primary thiolase due to its high enzymatic specificity towards the $C_{5}$ substrate. Given that $E$. coli has an endogenous thiolase (encoded by atoB), a deletion of atoB was expected to increase the ratio of $3 \mathrm{HV} / 3 \mathrm{HB}$ as AtoB has been shown to prefer to condense two molecules of acetyl-CoA instead of one propionyl-CoA and one acetyl-CoA [19]. However, our preliminary result showed that the recombinant HCT 11 with an atoB deletion behaved exactly as the recombinant HCT 10 , and the deletion in ato $B$ had essentially no effect on $3 \mathrm{HV}$ production (data not shown), implying that ato $B$ may not be a constitutively expressed gene. In addition, it is noteworthy that increased $3 \mathrm{HV}$ production in the recombinant HCT 20 relative to the recombinant HCT 10 was observed only with 2 -ketobutyrate supplementation (Figure 3) but not with the threonine supplementation, solely glucose, or solely glycerol experiments (Figure 4, 5 and 6); similarly, an accumulation of propionate only occurred in the 2-ketobutyrate supplementation experiment (Figure 3), altogether, indicating that $3 \mathrm{HV}$ biosynthesis from glucose or glycerol is most likely limited by the precursor propionyl-CoA. Therefore, approaches to increase the availability of propionyl-CoA could enhance the $3 \mathrm{HV}$ production.

\section{Conclusions}

Carbon skeletons with even-chain number are naturally found in fatty acid metabolism, but those with odd-chain number are pretty novel. As a result, there is a good deal of interest in making odd-carbon chain molecules such as $3 \mathrm{HV}(\mathrm{C} 5)$ and propionate $(\mathrm{C} 3)$ because they are so much harder to get to than even-carbon chain ones such as acetate (C2) and butyrate/butanol (C4). This paper opens the way for biosynthesis of the odd-carbon chain molecules from renewable feedstocks. Taking together, our work represents the first report of direct microbial production of enantiomerically pure $3 \mathrm{HV}$ from a single carbon source. In addition, we have explored the production of each stereoisomer of 3HV across different genetically altered $E$. coli strains, along with various enzyme homologs, for enhanced chiral $3 \mathrm{HV}$ production. Further engineering of host strains and pathway enzymes should lead to higher $3 \mathrm{HV}$ titers and a more economical bioprocess for the production of chiral $3 \mathrm{HV}$.

\section{Methods \\ Microorganisms}

The bacterial strains used are listed in Table 1. C. acetobutylicum ATCC 824, C. glutamicum ATCC 13032, and a threonine hyper-producer E. coli ATCC 21277 were purchased from the American Type Culture Collection (ATCC, Manassas, VA). R. eutropha $\mathrm{H} 16$ was provided by Professor Anthony Sinskey of the Department of Biology at the Massachusetts Institute of Technology (Cambridge, MA, USA). E. coli DH10B (Invitrogen, Carlsbad, CA) and ElectroTen-Blue (Stratagene, La Jolla, CA) were used for transformation of cloning reactions and propagation of all plasmids. MG1655 (kindly donated by Professor Gregory Stephanopoulos of the Department of Chemical Engineering at the Massachusetts Institute of Technology, USA) was used as the parental strain for genetic modification. Host gene deletions of endA, recA, atoDA, ackA-pta, poxB, tdh, met $A$, and ato $B$ were achieved with $\mathrm{P} 1$ transduction using the Keio collection strains as donor cells [39]. The kanamycin cassette was removed using plasmid pCP20 as described by Datsenko and Wanner [40] and the successfully constructed mutant strains were verified by colony PCR using appropriate primers. Strains carrying a $\lambda D E 3$ lysogen were constructed using a $\lambda D E 3$ Lysogenization Kit (Novagen, Darmstadt, Germany) for sitespecific integration of $\lambda \mathrm{DE} 3$ prophage into each host.

\section{Plasmid Construction}

Genes derived from C. acetobutylicum ATCC 824 ( $h b d$ and ptb-buk operon), R. eutropha $\mathrm{H} 16$ (bktB and phaB), C. glutamicum ATCC 13032 (ilvA), E. coli MG1655 (tesB, ilvA, and thrABC opeon), and E. coli ATCC 21277 ( $t h r A^{\mathrm{G} 1297 \mathrm{~A}} B C$ opeon) were obtained by polymerase chain reaction (PCR) using genomic DNA (gDNA) templates. All gDNAs were prepared using the Wizard Genomic DNA Purification Kit (Promega, Madison, 
Table 1 E. coli strains, plasmids and oligonucleotides used

\begin{tabular}{|c|c|c|}
\hline Name & Relevant Genotype & Reference \\
\hline \multicolumn{3}{|l|}{ Strains } \\
\hline $\mathrm{DH} 10 \mathrm{~B}$ & 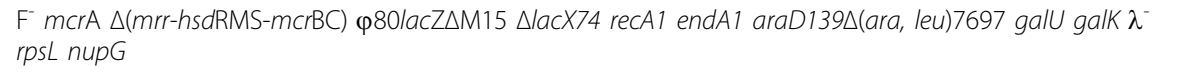 & Invitrogen \\
\hline ElectroTen-Blue & $\begin{array}{l}\Delta(m c r A) 183 \Delta(\text { mcrCB-hsdSMR-mrr) } 173 \text { endA1 supE44 thi-1 recA1 gyrA96 relA1 lac Kan' [F' proAB lacläZMM15 } \\
\text { Tn10 (Tet')] }\end{array}$ & Stratagene \\
\hline MG1655 & $F^{-} \lambda^{-} i l v G-r f b-50 r p h-1$ & ATCC 700926 \\
\hline HCT 10 & MG1655 $\triangle$ endA $\triangle r e c A(D E 3)$ & This study \\
\hline HCT 11 & MG1655 $\triangle$ endA $\triangle r e c A \triangle a t o B(D E 3)$ & This study \\
\hline HCT 20 & MG1655 $\triangle$ endA $\triangle a c k A-p t a \triangle a t o D A \triangle p o x B(D E 3)$ & This study \\
\hline HCT 21 & MG1655 $\triangle$ endA $\triangle a c k A-p t a \triangle a t o D A \Delta p o x B \Delta m e t A \Delta t d h(D E 3)$ & This study \\
\hline \multicolumn{3}{|l|}{ Plasmids } \\
\hline pETDuet-1 & ColE1(pBR322) ori, lacl, T7lac, Amp ${ }^{R}$ & Novagen \\
\hline pCDFDuet-1 & CloDF13 ori, lacl, T7lac, Strep ${ }^{R}$ & Novagen \\
\hline pCOLADuet-1 & COLA ori, lacl, T7lac, Kan ${ }^{R}$ & Novagen \\
\hline pET-B & pETDuet-1 harboring bktB from $R$. eutropha $\mathrm{H} 16$ & This study \\
\hline pET-PB-B ${ }^{a}$ & pETDuet-1 harboring ptb-buk operon from C. acetobutylicum ATCC 824, and bktB from R. eutropha H16 & This study \\
\hline $\mathrm{pCDF}-\mathrm{H}$ & pCDFDuet-1 harboring hbd from C. acetobutylicum ATCC 824 & This study \\
\hline pCDF-T-H ${ }^{a}$ & pCDFDuet-1 harboring tesB from E. coli MG1655, and hbd from C. acetobutylicum ATCC 824 & This study \\
\hline pCDF-P & pCDFDuet-1 harboring phaB from R. eutropha $\mathrm{H} 16$ & This study \\
\hline pCDF-T-P a & pCDFDuet-1 harboring tesB from E. coli MG1655, and phaB from R. eutropha $\mathrm{H} 16$ & This study \\
\hline pCOLA-lec & pCOLADuet-1 harboring ilvA from E. coli MG1655 & This study \\
\hline pCOLA-lcg & pCOLADuet-1 harboring ilvA from C. glutamicum & This study \\
\hline pCOLA-Tec-Icg ${ }^{a}$ & pCOLADuet-1 harboring thrABC operon from E. coli MG1655, and ilvA from C. glutamicum ATCC 13032 & This study \\
\hline pCOLA-Tecm-lcg a & $\begin{array}{l}\text { pCOLADuet-1 harboring thrA }{ }^{\mathrm{G} 1297 \mathrm{~A}} B C \text { operon from E. coli ATCC 21277, and ilvA from C. glutamicum } \\
\text { ATCC } 13032\end{array}$ & This study \\
\hline Primers $^{\mathbf{b}}$ & Sequence $5^{\prime} \rightarrow 3^{\prime c}$ & \\
\hline bktB_US_EcoRl & GAATTCATGACGCGTGAAGTGGTAGTG & Sigma-Genosys \\
\hline bktB_DS_Xhol & CTCGAGCGCAAGGCTAACCTCAGAT & Sigma-Genosys \\
\hline hbd_US_Ndel & ATTCATATGAAAAAGGTATGTGTTATAGG & Sigma-Genosys \\
\hline hbd_DS_Avrll & ATTCCTAGGCAGGTCGACTCTAGAACTTA & Sigma-Genosys \\
\hline phaB_US_Mfel & ATTCAATTGACGAAGCCAATCAAGGAG & Sigma-Genosys \\
\hline phaB_DS_Avrll & ATTCCTAGGGGTCAGCCCATATGCAG & Sigma-Genosys \\
\hline tesB_US_Ncol & ATTCCATGGGCATGAGTCAGGCGCTAA & Sigma-Genosys \\
\hline tesB_DS_Notl & ATTGCGGCCGCGACTCTAGAGACTTAATTGTG & Sigma-Genosys \\
\hline ilvAec_US_Ndel & ATTACATATGGCTGACTCGCAAC & Sigma-Genosys \\
\hline ilvAec_DS_Avrll & ATTACCTAGGCATITITCCCTAACC & Sigma-Genosys \\
\hline ilvAcg_US_Ndel & ATTACATATGAGTGAAACATACGTGTC & Sigma-Genosys \\
\hline ilvAcg_DS_Avrll & ATTACCTAGGCCTTCAGCTATGTTTA & Sigma-Genosys \\
\hline thrABC_US_BamHI & ATTAGGATCCAAGGAGATATATCATGCGAGTGTTGAAG & Sigma-Genosys \\
\hline thrABC_US_Ncol & ATTACCATGGGCATGCGAGTGTTGAAG & Sigma-Genosys \\
\hline thrABC_DS_Sall & ATTAGTCGACGATAATGAATAGATTTACTGATG & Sigma-Genosys \\
\hline
\end{tabular}

${ }^{a}$ Each gene is under the control of the T7lac promoter with a ribosome binding site.

b Primers were synthesized at Sigma-Genosys, St. Louis, MO.

c Restriction enzyme sites used in the cloning are shown in underlined italics.

WI). Custom oligonucleotides (primers) were purchased for all PCR amplifications (Sigma-Genosys, St. Louis, MO) as listed in Table 1. In all cases, Phusion High Fidelity DNA polymerase (Finnzymes, Espoo, Finland) was used for DNA amplification. Restriction enzymes and T4 DNA ligase were purchased from New England
Biolabs (Ipswich, MA). Recombinant DNA techniques were performed according to standard procedures [41]. Three co-replicable vectors, pETDuet-1, pCDFDuet-1, and pCOLADuet-1 (Novagen, Darmstadt, Germany), were used for construction of chiral $3 \mathrm{HV}$ biosynthetic pathways [42]. All vectors contain two multiple cloning 
sites (MCS), each of which is preceded by a T7lac promoter and a ribosome binding site (RBS), affording high-level expression of each individual gene.

Plasmids constructed in the present work are listed in Table 1. For cloning genes, PCR products incorporated with desired restriction sites within the 5 ' and 3 ' primers were digested, and the resulting DNA fragments were then cloned into pETDuet-1, pCDFDuet-1, or pCOLADuet-1. The $b k t B$ gene was inserted in between the $M f e \mathrm{I}$ and XhoI sites (MCS II) of pETDuet-1 to create pET-B. The $p t b-b u k$ gene, digested from pCDF-PB with EcoRI and NotI [15], was inserted between the EcoRI and NotI sites (MCS I) of pET-B to create pET-PB-B. Plasmid pCDF-H was created by inserting the $h b d$ gene between the NdeI and AvrII sites (MCS II) of pCDFDuet-1. Cloning the tesB gene between the NcoI and NotI sites (MCS I) of pCDFDuet-1 resulted in plasmid pCDF-T. Plasmid pCDF-T-H was then created by inserting the $h b d$ gene between the NdeI and AvrII sites (MCS II) of pCDF-T. In a similar manner, plasmid pCDF-P was created by inserting the $p h a B$ gene between the $M f e \mathrm{I}$ and $A v r \mathrm{II}$ sites (MCS II) of pCDFDuet-1. Plasmid pCDF-T-P was created by inserting the $p h a B$ gene between the $M f e \mathrm{I}$ and $A v r$ II sites (MCS II) of pCDF-T. Plasmids of pCOLA-Iec and pCOLA-Icg were constructed by inserting the E. coli ilvA and C. glutamicum ilvA, respectively, between the NdeI and AvrII sites (MCS II) of pCOLADuet-1. The thrABC operon from MG1655 was inserted in between the NcoI and SalI sites (MCS I) of pCOLADuet-1 to create pCOLA-Tec. Plasmid pCOLA-Tec-Icg was then created by inserting the C. glutamicum ilvA gene between the NdeI and AvrII sites (MCS II) of pCOLA-Tec. To construct plasmid pCOLA-Tecm-Icg, the $t h r A^{\mathrm{G} 1997 \mathrm{~A}} B C$ operon from E. coli ATCC 21277 was inserted in between the BamHI and SalI sites (MCS I) of pCOLA-Icg. All constructs were confirmed to be correct by restriction enzyme digestion and nucleotide sequencing.

\section{Culture Conditions}

Seed cultures of the recombinant strains were grown in LB medium at $30^{\circ} \mathrm{C}$ overnight on a rotary shaker at 250 $\mathrm{rpm}$. For the biosynthesis of chiral $3 \mathrm{HV}$, the seed cultures were used to inoculate $50 \mathrm{~mL}$ LB medium supplemented with $20 \mathrm{~g} / \mathrm{L}$ glucose or $20 \mathrm{~g} / \mathrm{L}$ glycerol at an inoculation volume of $2 \%$ in $250 \mathrm{~mL}$ flasks. Cultures were then incubated at $30^{\circ} \mathrm{C}$ on a rotary shaker until $\mathrm{OD}_{600}$ reached 0.8 1.0. At this point, $1 \mathrm{mM}$ IPTG was added to the cultures to induce recombinant protein expression. Following induction, cells were cultivated at $30^{\circ} \mathrm{C}$ and sampled at $24 \mathrm{~h}$ intervals for up to $72 \mathrm{~h}$ postinduction for HPLC analysis. We found that both $3 \mathrm{HB}$ and 3HV titers did not reach a plateau until $48 \mathrm{~h}$ and that there was essentially no difference in the titers between $48 \mathrm{~h}$ and $72 \mathrm{~h}$. Accordingly, only the peak titers observed at $48 \mathrm{~h}$ were reported in this study. In some experiments as indicated, $20 \mathrm{mM}(\sim 1.92 \mathrm{~g} / \mathrm{L})$ sodium propionate, $3 \mathrm{~g} / \mathrm{L}$ sodium 2-ketobutyrate, or $3 \mathrm{~g} / \mathrm{L}$ threonine was added into the cultures at the same time of induction. In all cases, LB medium was supplemented with $50 \mathrm{mg} / \mathrm{L}$ ampicillin, $50 \mathrm{mg} / \mathrm{L}$ streptomycin, and $30 \mathrm{mg} / \mathrm{L}$ kanamycin, as appropriate. In general, experiments were performed in triplicates, and data are presented as the averages and standard deviations of the results.

\section{Metabolite Analysis}

Samples were centrifuged to pellet cells while the aqueous supernatant was collected for HPLC analysis. Products of interest, including $3 \mathrm{HB}, 3 \mathrm{HV}$, glucose, glycerol, 2-ketobutyrate, acetate, and propionate, were analyzed via HPLC using an Agilent 1100 series instrument equipped with a refractive index detector (RID) and a diode array detector (DAD). Given that the 3HB peak is overlapped with the glycerol peak in the RID chromatogram, detection of $3 \mathrm{HB}$ in the glycerol-fed cultures was achieved using the DAD at $210 \mathrm{~nm}$. Analyte separation was achieved using an Aminex ${ }^{\circ} \mathrm{HPX}-87 \mathrm{H}$ anion exchange column (Bio-Rad Laboratories, Hercules, CA) with $5 \mathrm{mM} \mathrm{H}_{2} \mathrm{SO}_{4}$ as the mobile phase. The mobile phase was pumped at a constant rate of $0.6 \mathrm{~mL} / \mathrm{min}$, and the column and detector temperatures were each set at $35^{\circ} \mathrm{C}$ throughout. Concentrations were determined by linear extrapolation from calibration of external standards.

\section{Chiral Analysis of 3HV}

The stereochemistry of $3 \mathrm{HV}$ produced was determined by methyl esterification of the $3 \mathrm{HV}$ present in the medium followed by chiral HPLC analysis as described in a previously reported method [15]. The chiral analysis was performed on an Agilent 1100 Series instrument equipped with a Chiralcel OD-H column $(0.46 \mathrm{~cm} \varphi \times 25$ $\mathrm{cm}$ ) purchased from Daicel Chemical Industries (West Chester, PA). Methyl-3HV was detected on a DAD at $210 \mathrm{~nm}$. The mobile phase was 9:1 $n$-hexane:isopropanol and the flow rate through the column was $0.7 \mathrm{~mL} / \mathrm{min}$. Due to unavailability of standards of Methyl- $(R)-3 \mathrm{HV}$ and Methyl- $(S)-3 \mathrm{HV}$, these spectra were compared to a racemic 3HV standard (Epsilon Chimie, Brest, France) derivatized by methyl esterification.

\section{Acknowledgements}

We acknowledge financial support by the Synthetic Biology Engineering Research Center (SynBERC) funded by the National Science Foundation (Grant EEC-0540879), the MIT Energy Initiative (MITEI), and Shell Global Solutions (US) Inc.

\section{Author details}

${ }^{1}$ Department of Chemical Engineering, Massachusetts Institute of Technology, Cambridge, MA 02139, USA. Synthetic Biology Engineering 
Research Center (SynBERC), Massachusetts Institute of Technology, Cambridge, MA 02139, USA. ${ }^{3}$ Dow Chemical Company, Spring House, PA 19477. USA

\section{Authors' contributions}

HCT and KLJP initiated and coordinated the project. HCT performed experiments. CLH assisted HCT with gene cloning, cell culture, and data analysis. CHM performed the chiral analysis. HCT wrote and KLJP edited the paper. All authors approved the final version of the manuscript.

\section{Competing interests}

The authors declare that they have no competing interests.

Received: 28 September 2010 Accepted: 27 November 2010 Published: 27 November 2010

\section{References}

1. Patel RN: Stereoselective Biocatalysis Boca Raton, FL.: CRC Press; 2000

2. Tokiwa Y, Calabia BP: Biological production of functional chemicals from renewable resources. Can J Chem 2008, 86:548-555.

3. Shiraki M, Endo T, Saito T: Fermentative production of $(R)-(-)-3-$ hydroxybutyrate using 3-hydroxybutyrate dehydrogenase null mutant of Ralstonia eutropha and recombinant Escherichia coli. J Biosci Bioeng 2006, 102:529-534

4. Chen GQ, Wu Q: Microbial production and applications of chiral hydroxyalkanoates. Appl Microbiol Biotechnol 2005, 67:592-599.

5. Zhao K, Tian G, Zheng Z, Chen JC, Chen GQ: Production of D-(-)-3hydroxyalkanoic acid by recombinant Escherichia coli. FEMS Microbiol Lett 2003, 218:59-64

6. Ren $\mathrm{Q}$, Ruth $\mathrm{K}$, Th?ny-Meyer L, Zinn M: Enatiomerically pure hydroxycarboxylic acids: current approaches and future perspectives. Appl Microbiol Biotechnol 2010, 87(1):41-52.

7. Chiba T, Nakai T: A new synthetic approach to the carbapenem antibiotic PS-5 from ethyl(S)-3-hydroxybutanoate. Chem Lett 1987, 11:2187-2188.

8. Seebach D, Chow HF, Jackson RFW, Sutter MA, Thaisrivongs $S_{\text {, }}$ Zimmermann J: (+)-11,11'-di-O-methylelaiophylidene-preparation from Elaiophylin and total synthesis from (R)-3-hydroxybutyrate and (S)malate. Liebigs Ann Chem 1986, 1986:1281-1308.

9. Chiba T, Nakai TA: Synthetic approach to (1)-thienamycin from methyl (R)-(2)-3-hydroxybutanoate. A new entry to $(3 R, 4 R)-3-[(R)-1-$ hydroxyethyl]-4-acetoxy-2-azetidinone. Chem Lett 1985, 161:651-654.

10. Mori K: A simple synthesis of (S)-(+)-sulcatol, the pheromone of Gnathotrichus tetusus employing baker's yeast for asymmetric reduction. Tetrahedron 1981, 37:1341-1342.

11. Steinbuchel A, Valentin HE: Diversity of bacterial polyhydroxyalkanoic acids. FEMS Microbiology Letters 1995, 128:219-228.

12. Liu Q, Ouyang SP, Chung A, Wu Q, Chen GQ: Microbial production of $R$-3hydroxybutyric acid by recombinant $E$. coli harboring genes of $p h b A$ phbB, and tesB. Appl Microbiol Biotechnol 2007, 76:811-818.

13. Lee SH, Park SJ, Lee SY, Hong SH: Biosynthesis of enantiopure (S)-3hydroxybutyric acid in metabolically engineered Escherichia coli. Appl Microbiol Biotechnol 2008, 79:633-641.

14. Lee SY, Lee Y: Metabolic engineering of Escherichia coli for production of enantiomerically pure (R)-(-)-hydroxycarboxylic acids. Appl Environ Microbiol 2003, 69:3421-3426.

15. Tseng HC, Martin CH, Nielsen DR, Prather KL: Metabolic engineering of Escherichia coli for enhanced production of (R)- and (S)-3hydroxybutyrate. Appl Environ Microbiol 2009, 75:3137-3145.

16. Hasegawa J HS, Ogura M, Watanabe K: Production of betahydroxycarboxylic acids from aliphatic carboxylic acids by microorganisms. J Ferment Technol 1981, 59:257-262

17. Bramucci MG, Dicosimo, Robert, Fallon, Robert, Gavagan, John E Herkes, Frank, Wilczek, Lech : 3-Hydroxycarboxylic acid production and use in branched polymers. United States Patent 71384802006.

18. Martin $\mathrm{CH}$, Prather $\mathrm{KLJ}$ : High-titer production of monomeric hydroxyvalerates from levulinic acid in Pseudomonas putida. Journal of Biotechnology 2009, 139:61-67.

19. Slater S, Houmiel KL, Tran M, Mitsky TA, Taylor NB, Padgette SR, Gruys KJ: Multiple beta-ketothiolases mediate poly(beta-hydroxyalkanoate) copolymer synthesis in Ralstonia eutropha. J Bacteriol 1998, 180:1979-1987.
20. Eschenlauer AC, Stoup SK, Srienc F, Somers DA: Production of heteropolymeric polyhydroxyalkanoate in Escherichia coli from a single carbon source. Int J Biol Macromol 1996, 19:121-130.

21. Poirier $Y$, Nawrath C, Somerville C: Production of polyhydroxyalkanoates, a family of biodegradable plastics and elastomers, in bacteria and plants. Biotechnology (N Y) 1995, 13:142-150.

22. Aldor IS, Kim SW, Prather KL, Keasling JD: Metabolic engineering of a novel propionate-independent pathway for the production of poly(3hydroxybutyrate-co-3-hydroxyvalerate) in recombinant Salmonella enterica serovar typhimurium. Appl Environ Microbiol 2002, 68:3848-3854.

23. Madison LL, Huisman GW: Metabolic engineering of poly(3hydroxyalkanoates): from DNA to plastic. Microbiol Mol Biol Rev 1999 63:21-53.

24. Slater S, Gallaher T, Dennis D: Production of poly-(3-hydroxybutyrate-co-3hydroxyvalerate) in a recombinant Escherichia coli strain. Appl Environ Microbiol 1992, 58:1089-1094.

25. Liu SJ, Steinbuchel A: Exploitation of butyrate kinase and phosphotransbutyrylase from Clostridium acetobutylicum for the in vitro biosynthesis of poly(hydroxyalkanoic acid). Appl Microbiol Biotechnol 2000, 53:545-552.

26. Bisswanger $\mathrm{H}$ : Substrate specificity of the pyruvate dehydrogenase complex from Escherichia coli. J Biol Chem 1981, 256:815-822

27. Morbach S, Sahm H, Eggeling L: I-Isoleucine Production with Corynebacterium glutamicum: Further Flux Increase and Limitation of Export. Appl Environ Microbiol 1996, 62:4345-4351.

28. Lee JH, Sung BH, Kim MS, Blattner FR, Yoon BH, Kim JH, Kim SC: Metabolic engineering of a reduced-genome strain of Escherichia coli for Lthreonine production. Microb Cell Fact 2009, 8:2

29. Murarka A, Dharmadi Y, Yazdani SS, Gonzalez R: Fermentative utilization of glycerol by Escherichia coli and its implications for the production of fuels and chemicals. Appl Environ Microbiol 2008, 74:1124-1135.

30. Gonzalez-Pajuelo M, Andrade JC, Vasconcelos I: Production of 1,3propanediol by Clostridium butyricum VPI 3266 using a synthetic medium and raw glycerol. J Ind Microbiol Biotechnol 2004, 31:442-446.

31. Shams Yazdani S, Gonzalez R: Engineering Escherichia coli for the efficient conversion of glycerol to ethanol and co-products. Metab Eng 2008 10:340-351.

32. Lee HC, Kim JS, Jang W, Kim SY: Thymidine production by overexpressing NAD+ kinase in an Escherichia coli recombinant strain. Biotechnol Lett 2009, 31:1929-1936.

33. Debabov VG: The threonine story. Adv Biochem Eng Biotechnol 2003, 79:113-136.

34. Lee KH, Park JH, Kim TY, Kim HU, Lee SY: Systems metabolic engineering of Escherichia coli for L-threonine production. Mol Syst Biol 2007, 3:149.

35. Conrado RJ, Varner JD, DeLisa MP: Engineering the spatial organization of metabolic enzymes: mimicking nature's synergy. Curr Opin Biotechnol 2008, 19:492-499.

36. Moll JR, Ruvinov SB, Pastan I, Vinson C: Designed heterodimerizing leucine zippers with a ranger of pls and stabilities up to 10(-15) M. Protein Sci 2001, 10:649-655.

37. Dueber JE, Wu GC, Malmirchegini GR, Moon TS, Petzold CJ, Ullal AV, Prather KL, Keasling JD: Synthetic protein scaffolds provide modular control over metabolic flux. Nat Biotechnol 2009, 27:753-759.

38. Terpe K: Overview of bacterial expression systems for heterologous protein production: from molecular and biochemical fundamentals to commercial systems. Appl Microbiol Biotechnol 2006, 72:211-222.

39. Baba T, Ara T, Hasegawa M, Takai Y, Okumura Y, Baba M, Datsenko KA, Tomita M, Wanner BL, Mori H: Construction of Escherichia coli K-12 inframe, single-gene knockout mutants: the Keio collection. Mol Syst Biol 2006, 2, 20060008

40. Datsenko KA, Wanner BL: One-step inactivation of chromosomal genes in Escherichia coli K-12 using PCR products. Proc Natl Acad Sci USA 2000, 97:6640-6645.

41. Sambrook J, Russell D: Molecular Cloning: A Laboratory Manual. Third edition. Cold Spring Harbor, NY.: Cold Spring Harbor Laboratory Press; 2001.

42. Tolia NH, Joshua-Tor L: Strategies for protein coexpression in Escherichia coli. Nat Methods 2006, 3:55-64

43. Abramson J, Riistama S, Larsson G, Jasaitis A, Svensson-Ek M, Laakkonen L, Puustinen A, Iwata S, Wikstrom M: The structure of the ubiquinol oxidase from Escherichia coli and its ubiquinone binding site. Nat Struct Biol 2000, 7:910-917 
44. Yeh Jl, Chinte U, Du S: Structure of glycerol-3-phosphate dehydrogenase, an essential monotopic membrane enzyme involved in respiration and metabolism. Proc Natl Acad Sci USA 2008, 105:3280-3285.

doi:10.1186/1475-2859-9-96

Cite this article as: Tseng et al:: Biosynthesis of chiral 3-hydroxyvalerate from single propionate-unrelated carbon sources in metabolically engineered E. coli. Microbial Cell Factories 2010 9:96.

Submit your next manuscript to BioMed Central and take full advantage of:

- Convenient online submission

- Thorough peer review

- No space constraints or color figure charges

- Immediate publication on acceptance

- Inclusion in PubMed, CAS, Scopus and Google Scholar

- Research which is freely available for redistribution

Submit your manuscript at www.biomedcentral.com/submit
C Biomed Central 